
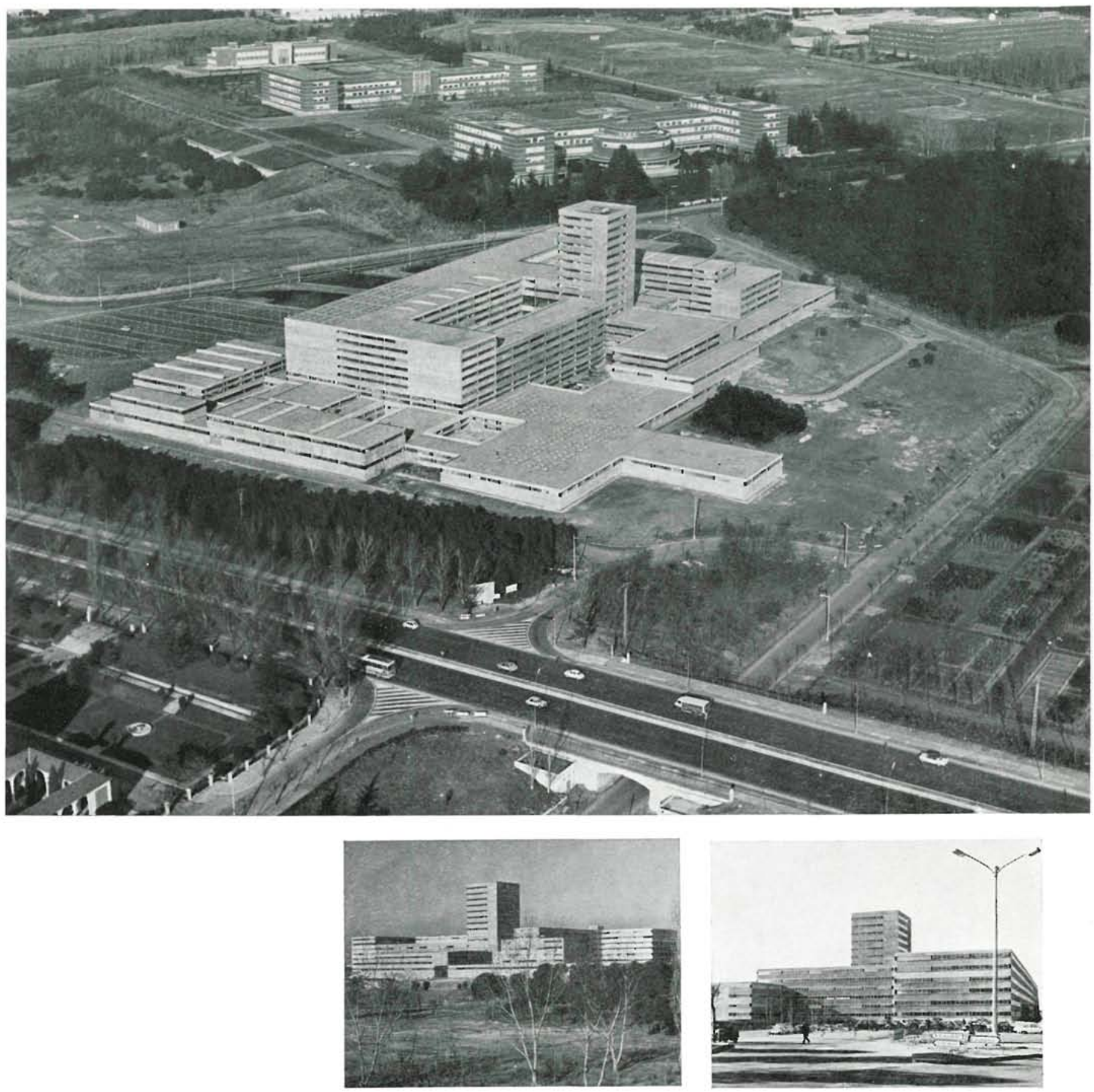

\title{
escuela técnica superior de ingenieros de caminos, canales y puertos, en Madrid - España
} LUIS LAORGA y JOSE LOPEZ ZANON, arquitectos

$142-73$

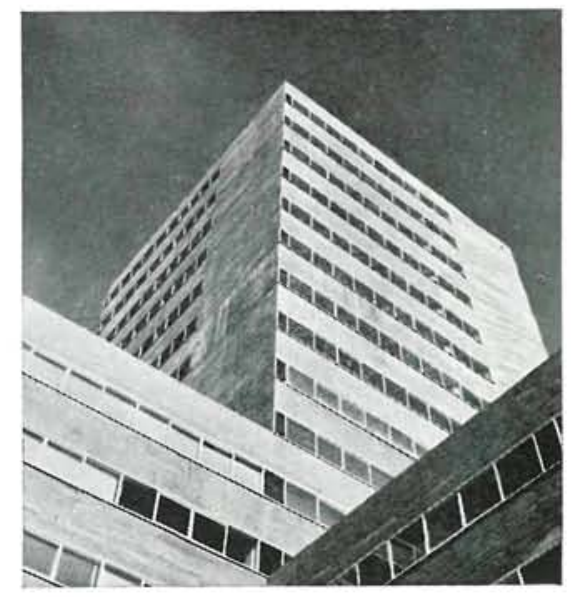



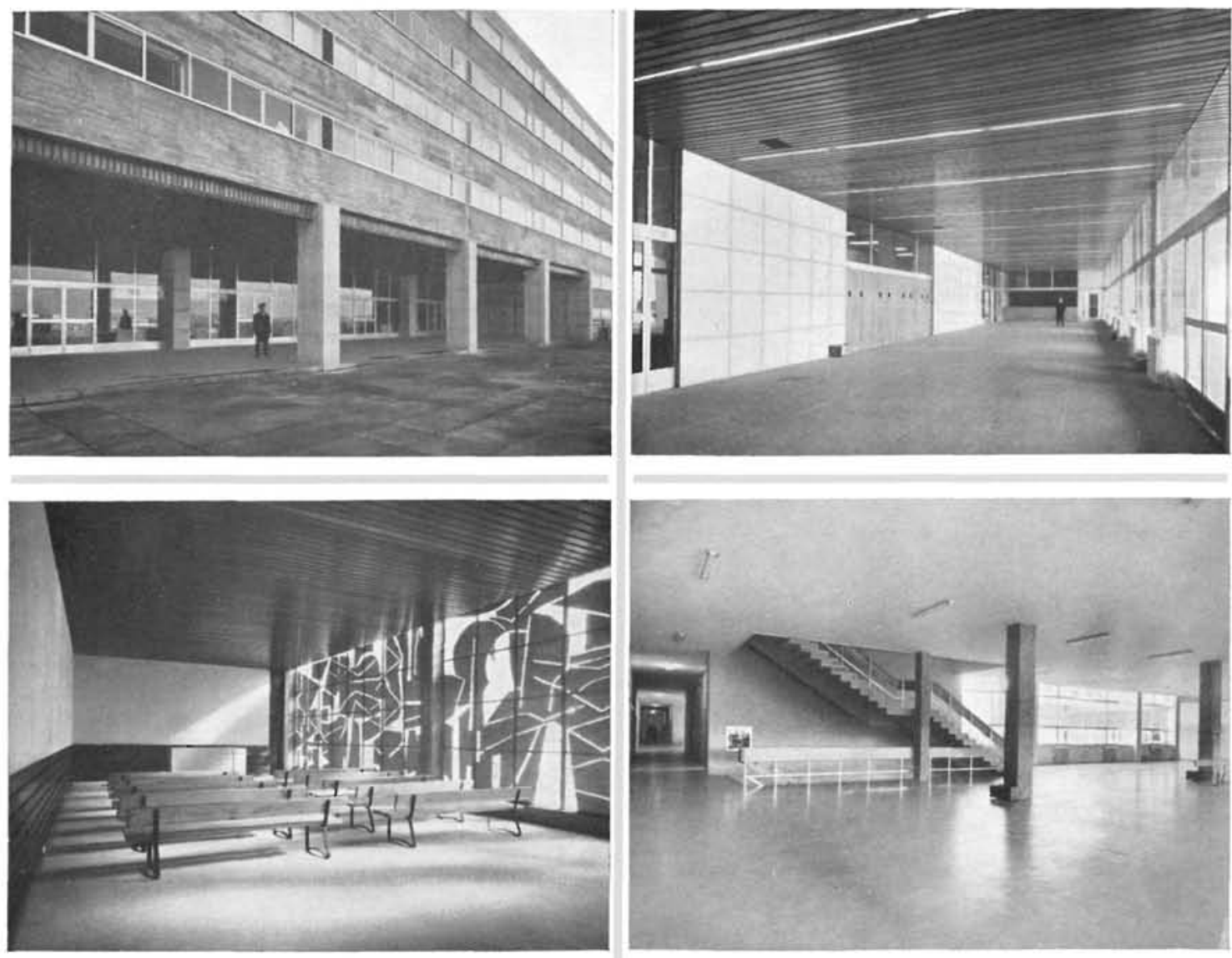

\section{sinm grosis}

El proyecto fue el primer premio de un concurso convocado para este fin en 1963 y está resuelto teniendo en cuenta las normas universalmente aceptadas de: dimensionamiento correcto y ajustado, iluminación uniforme, aireación transversal, reducción de plantas, concentración máxima y circulaciones cortas, organización jerárquica de los diferentes nú cleos y funciones, centralización de las áreas de relajación respecto de sus correspondien. tes grupos...

El edificio ha sido el resultado de una construcción cuidada y en la cual se han utilizado, fundamentalmente, materiales duraderos y de fácil conservación, tales como el hormigón visto $\mathbf{y}$ el aluminio anodizado.
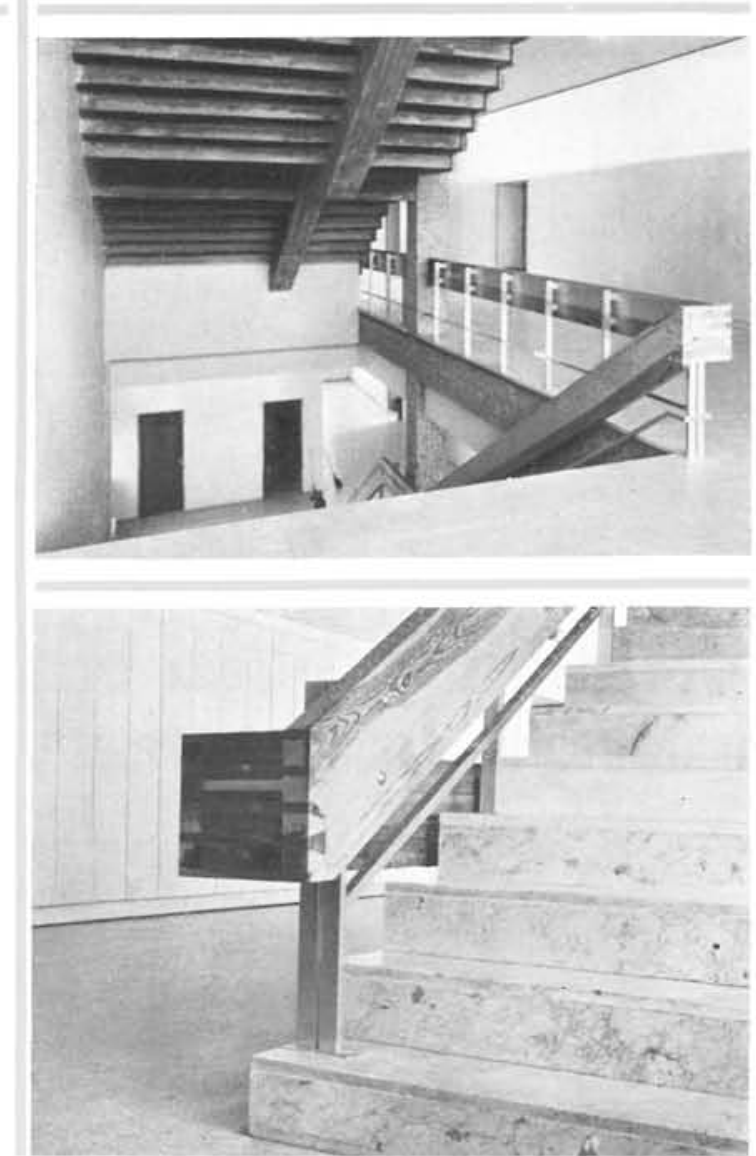


\section{Introducción}

El proyecto y construcción de edificios dedicados a la enseñanza tiene técnica específica en continua evolución, paralela a los avances de los métodos didácticos en que se apoya.

La Arquitectura escolar nace en el momento en que se abandona la imitación de los estilos históricos y se toma nueva orientación al principio, casi exclusivamente basada en la solución de los problemas de distribución, orientación, ventilación e iluminación.

Hoy día, dado el volumen e importancia de la obra ya experimentada, estamos en condiciones de hacer una revisión de la misma, sopesando las ventajas e inconvenientes que sus diversas soluciones presentan, teniendo en cuenta clima, economía y régimen del suelo.

En particular, la luminosidad del cielo de Madrid hace perder valor a la preocupación generalizada de utilizar grandes superficies acristaladas. Ello estriba en que con factores de iluminación (F. I.) de $5 \%$ pueden alcanzarse los 200 luxes, tope a partir del cual se produce fatiga visual y, como consecuencia, pérdida en el grado de atención. Se repite con frecuencia el caso paradójico de encarecer la obra con grandes ventanales para tener luego absoluta necesidad de tamizar el brillo con cortinas o persianas. Además, los huecos excesivos acarrean problemas de aislamiento fónico y térmico, especialmente en la meseta de clima extraordinariamente duro.

Como planeamiento general nos inclinamos decididamente por la idea de las realizaciones más recientes, basadas en el principio de las Plataformas, que resuelve el problema de circulaciones más cortas, permite dar forma arquitectónica a grupos complejos de edificios y que, además, está inspirada en las maravillosas enseñanzas de la Arquitectura tradicional de los conjuntos españoles.

En este camino se encuentran alineados Jorn Utzon, pionero de estas investigaciones; Henning Larsen, con el proyecto de la nueva Universidad de Estocolmo, y Roland Gross, con sus escuelas en Suiza.

\section{punto de partida}

El proyecto está resuelto con plantas concentradas, teniendo presentes las premisas aceptadas universalmente de:

Perfecto dimensionamiento en aulas, laboratorios y restantes locales.

Iluminación uniforme, de nivel apropiado, en dichos elementos.

Aireación transversal de los mismos.

Disposición constructiva flexible para permitir la extensibilidad y variabilidad de los espacios ante programas de enseñanza en evolución.

Reducción del número de plantas a un máximo de cuatro.

Organización jerárquica de los núcleos de diferente función, con situación preponderante de los más importantes y baricéntrica de los de usos comunes y generales.

Además de estas premisas se han tenido en cuenta las consideraciones específicas del caso, que estimamos en:

Concentración máxima, para conseguir el mayor ahorro en superficies construidas, fachadas, pérdidas térmicas, cubiertas y recorridos. Al propio tiempo que se defiende en lo posible el arbolado que se reserva para zona de aislamiento de la vía de tránsito rápido y se ahorra suelo para futuras necesidades.

Nucleación programada por cursos y actividades.

Disposición de las áreas de relajación de los diversos núcleos de manera que sobre ellas se abran los departamentos de actividad de los distintos grupos, e integradas todas ellas en el sistema circulatorio general.

Conseguir para cada escalón de actividad su propio camino dotado de sus accesos y sólo conectado con los otros escalones en los puntos de actuación común. 
planta primera

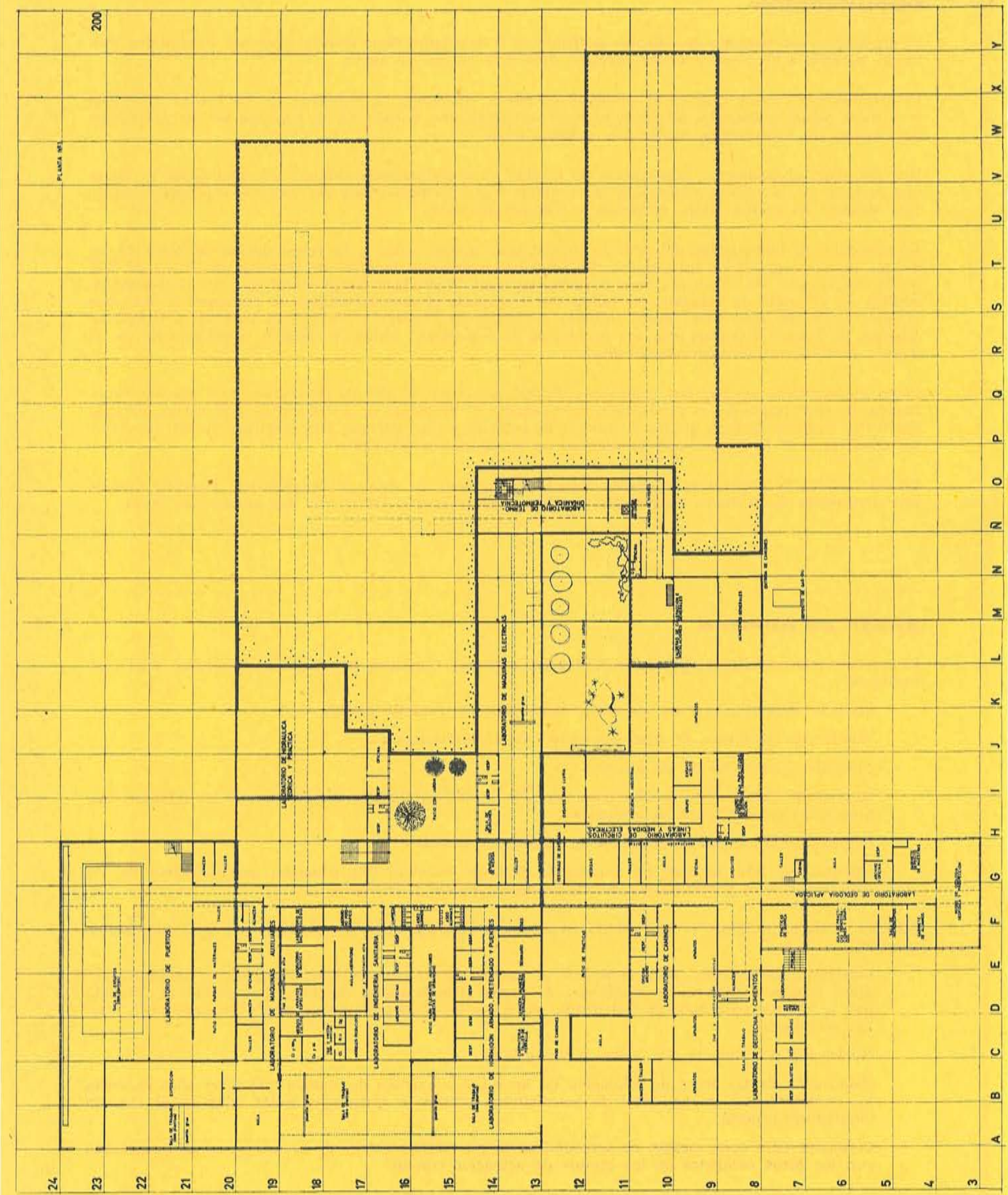


planta segunda

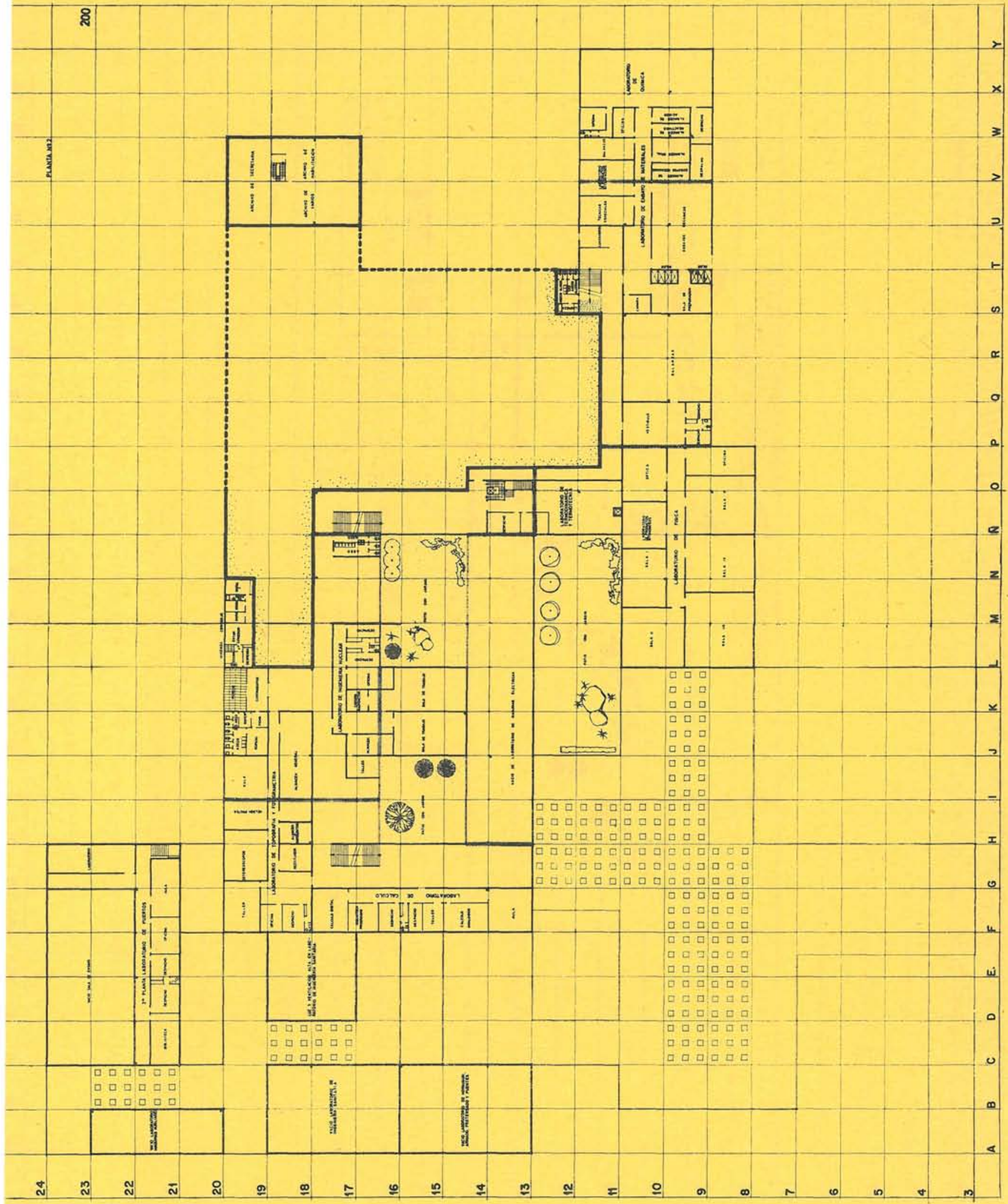


planta tercora

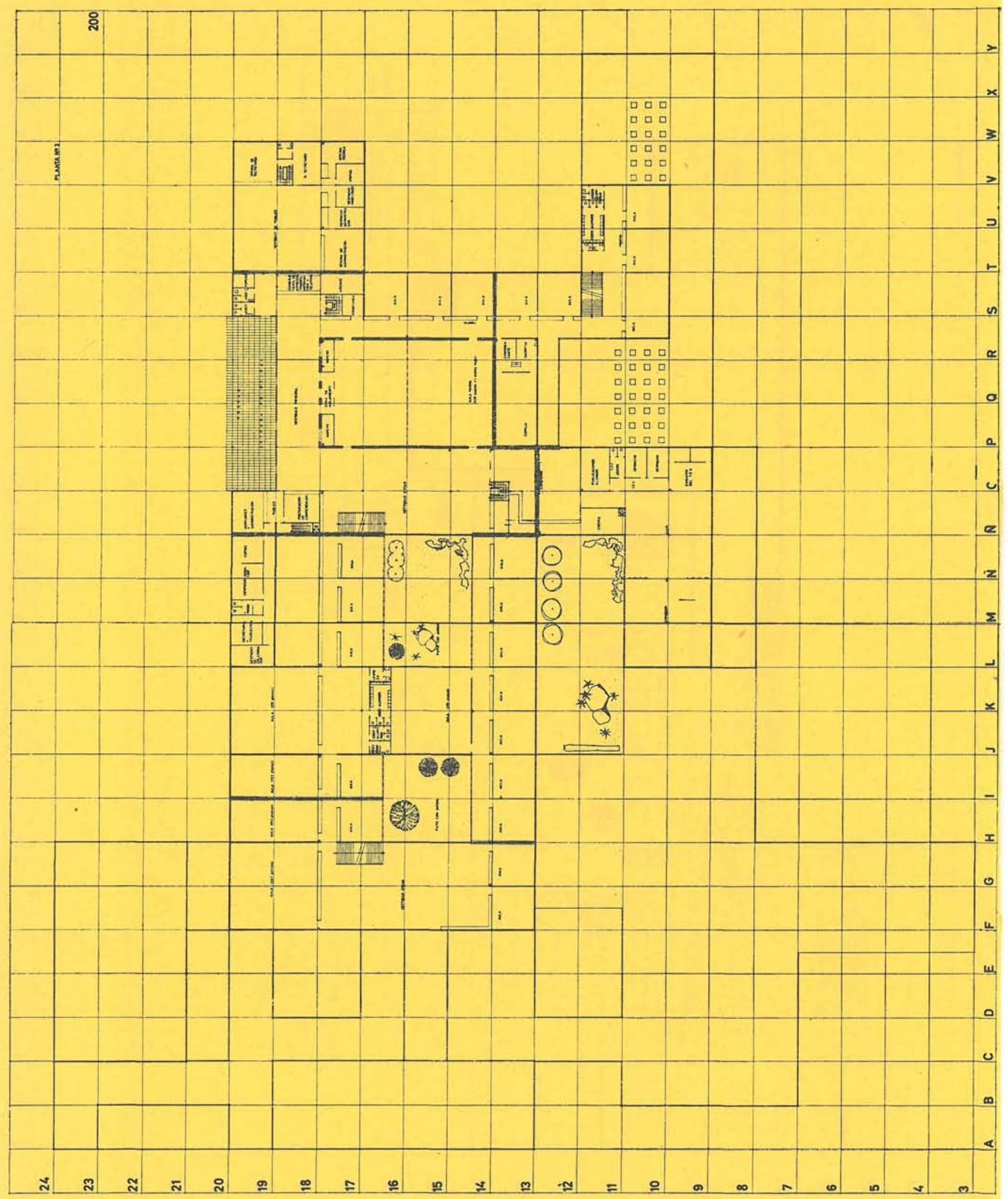

40 
planfa cuarta

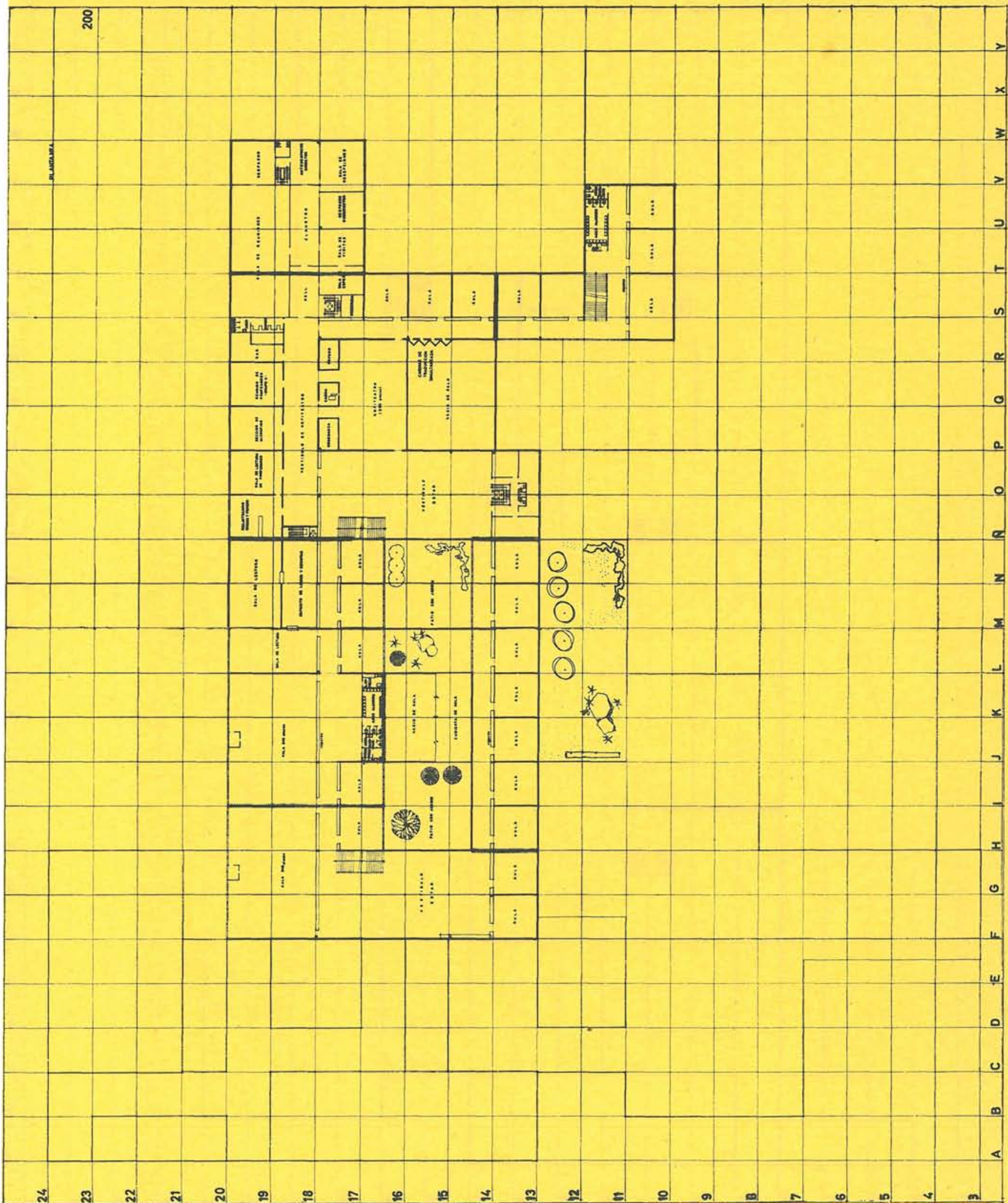


planta quinta

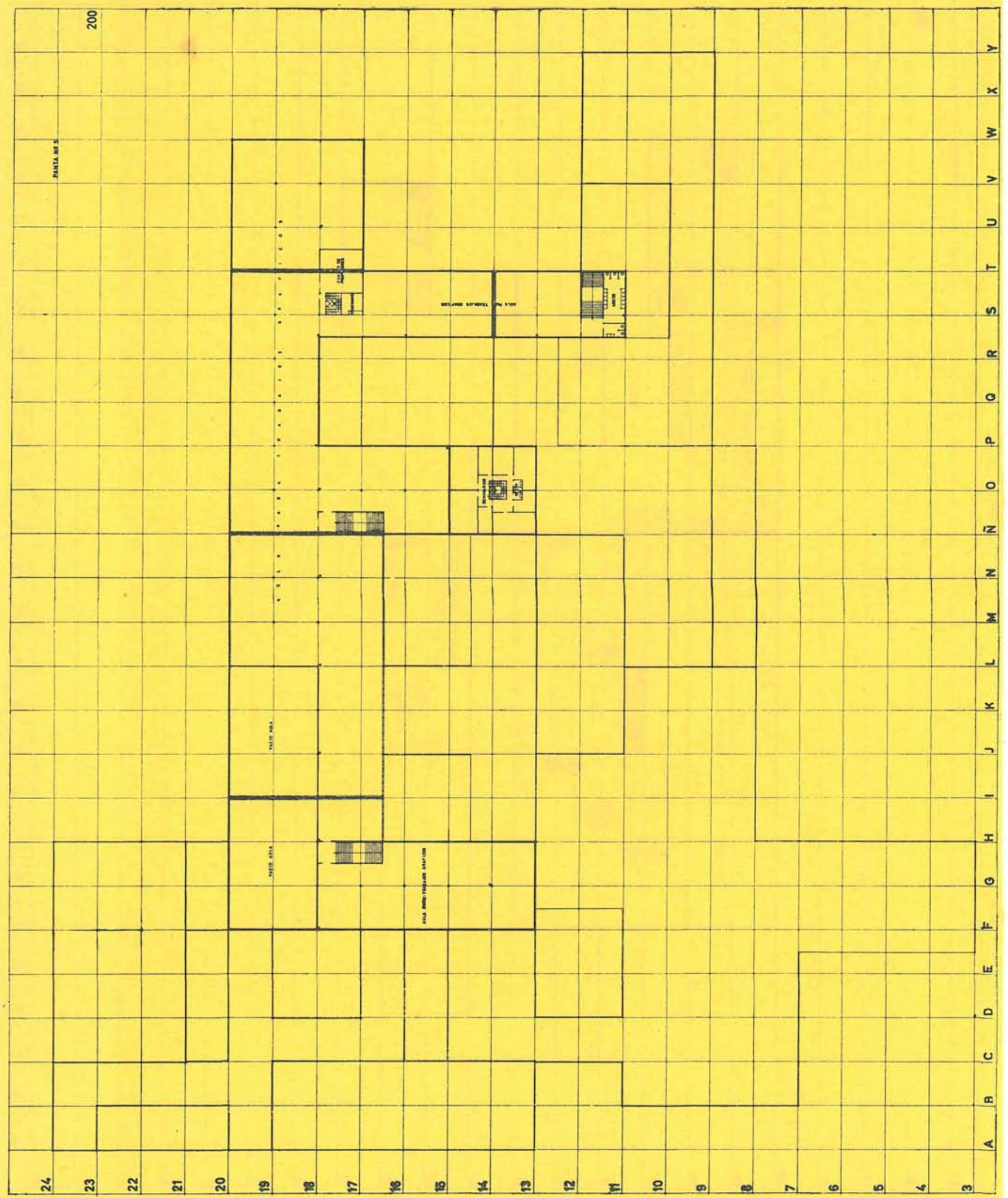




\section{essequeme}

Cuando el programa a desarrollar es complicado, como en nuestro caso, el esquema no puede reducirse a conjuntar una determinada colección de elementos conocidos. El problema puede resolverse eligiendo entre dos líneas de actuación claras y distantes y una tercera intermedia que resumimos así:

a) Solución de los diferentes problemas individuales de una forma también individual y una posterior yuxtaposición de las soluciones parciales para conseguir un resultado final.

b) Afrontar la solución de una manera global, considerando el problema en su máxima amplitud e intentando resolverlo también de forma unitaria.

c) Otra, entre las dos anteriores, que busca esta unidad final por la vía de la unidad o integración formal.

El primer camino supondría iniciar la materialización antes de haber llegado a establecer una verdadera unidad de base, y lo más que podríamos alcanzar con él sería la conjunción ingeniosa de un grupo de soluciones parciales.

El segundo, teóricamente el más correcto, supondría producir una síntesis del edificio antes de iniciar el esquema y sería llegar a sentirlo como un mecanismo único, un organismo complejo que solucionaría de forma homogénea y total el problema, sin reducirlo a sus elementos mínimos. Consistiría, en una palabra, en superar la idea de agrupación de elementos para llegar a lo que pudiéramos llamar el esquema integral.
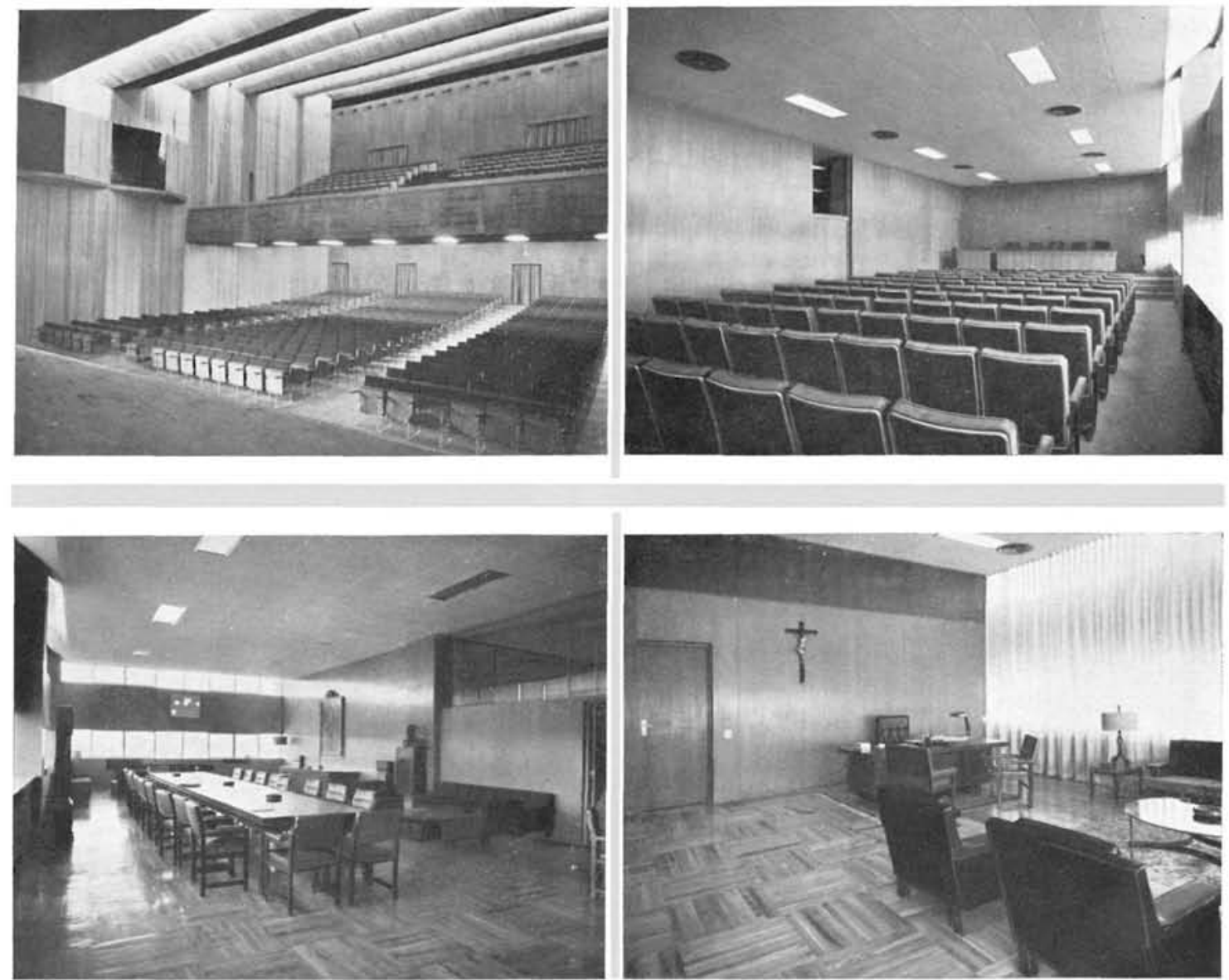
El tercero que hemos elegido aplica esta solución en su última fase, al resolver parcialmente los problemas dentro de una organización modular única. La defensa de la decisión no ofrece dudas; no es lícita la realización de un centro educacional que va a albergar enseñanzas con planes de estudio en evolución, con proyectos de criterio generativo dedicados en exclusividad a determinado plan.

\section{flexilbilidar}

A nuestro juicio, es una condición indispensable que debe de reunir cualquier edificio escolar. Es fundamental acomodarse a condiciones cambiantes con poco gasto.

Tal flexibilidad no debe alcanzar exclusivamente al elemento estructural, sino que debe de marcarse en planta.

Hemos considerado también imprescindible dejar crecedera la construcción en las direcciones de mayores posibilidades.

\section{ongumización del edificio}

En el bloque dominante se sitúa un núcleo bien definido de locales, en torno al vestíbulo principal; entre ellos se encuentran: los de uso general y recepción, administración, claustro y servicios de extensión. Corresponden a la planta noble: la dirección y biblioteca; a la baja: la administración y secretaría en contacto con la calle; y la última planta, conservada diáfana en su totalidad, se dedicará a funciones docen tes varias de gran volumen de alumnado.
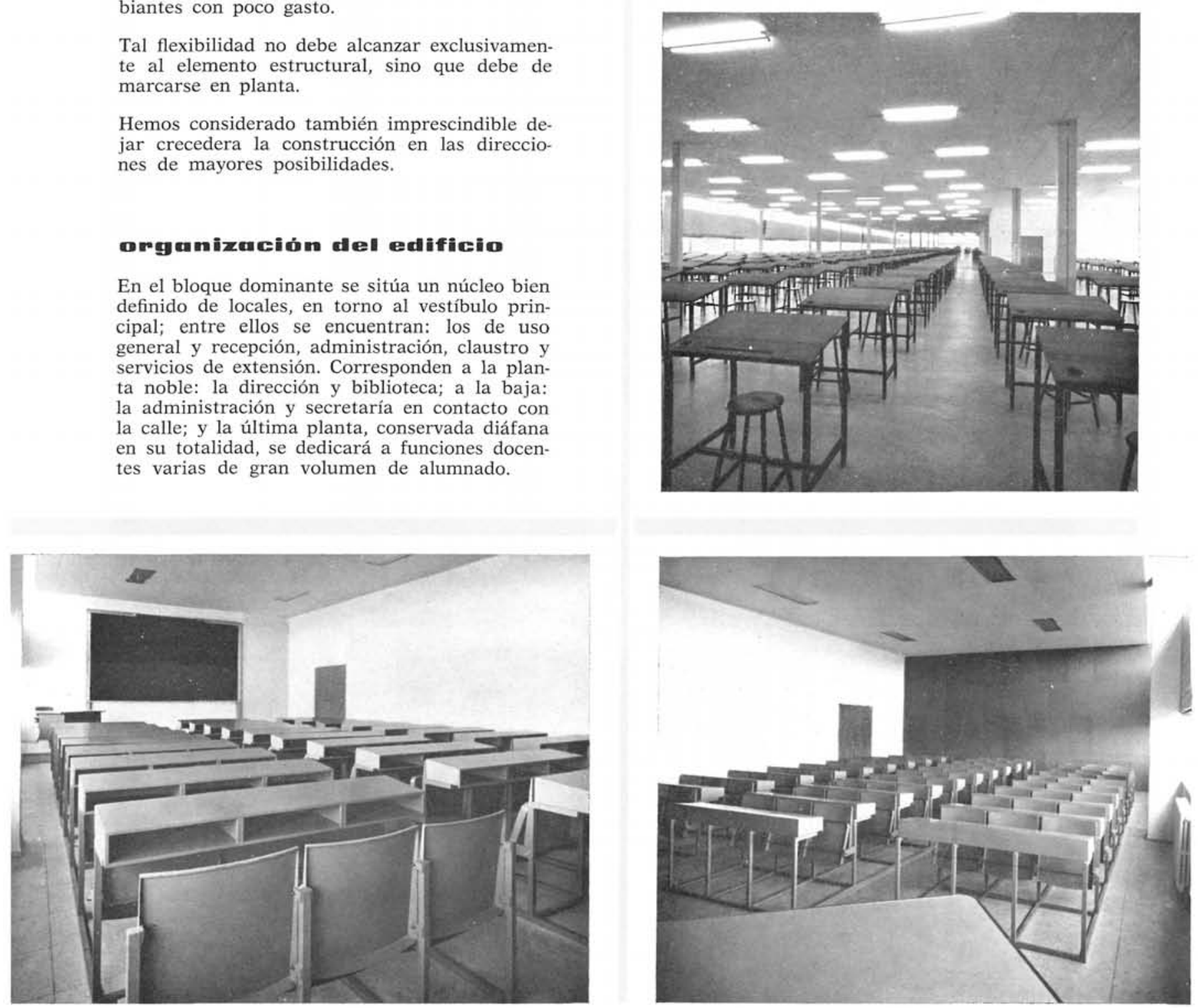

\section{clases}

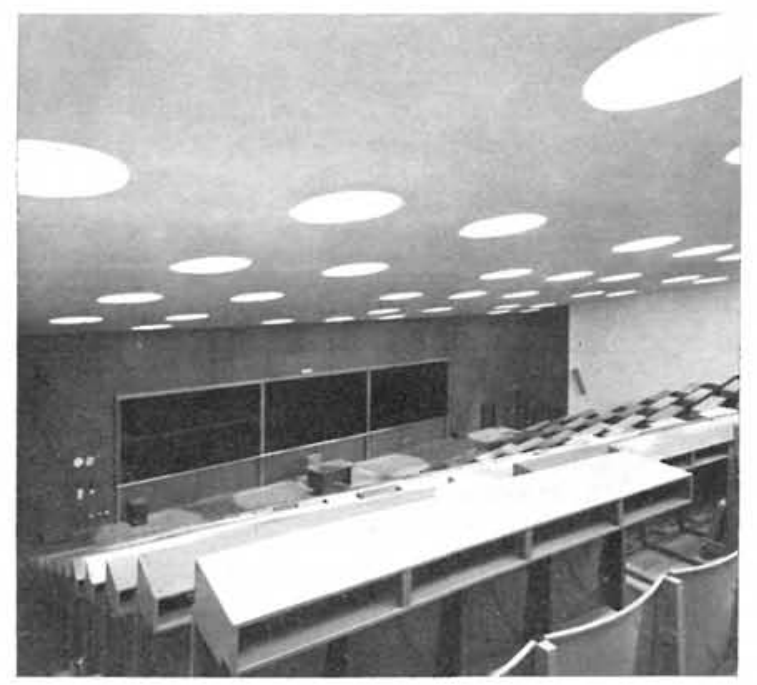



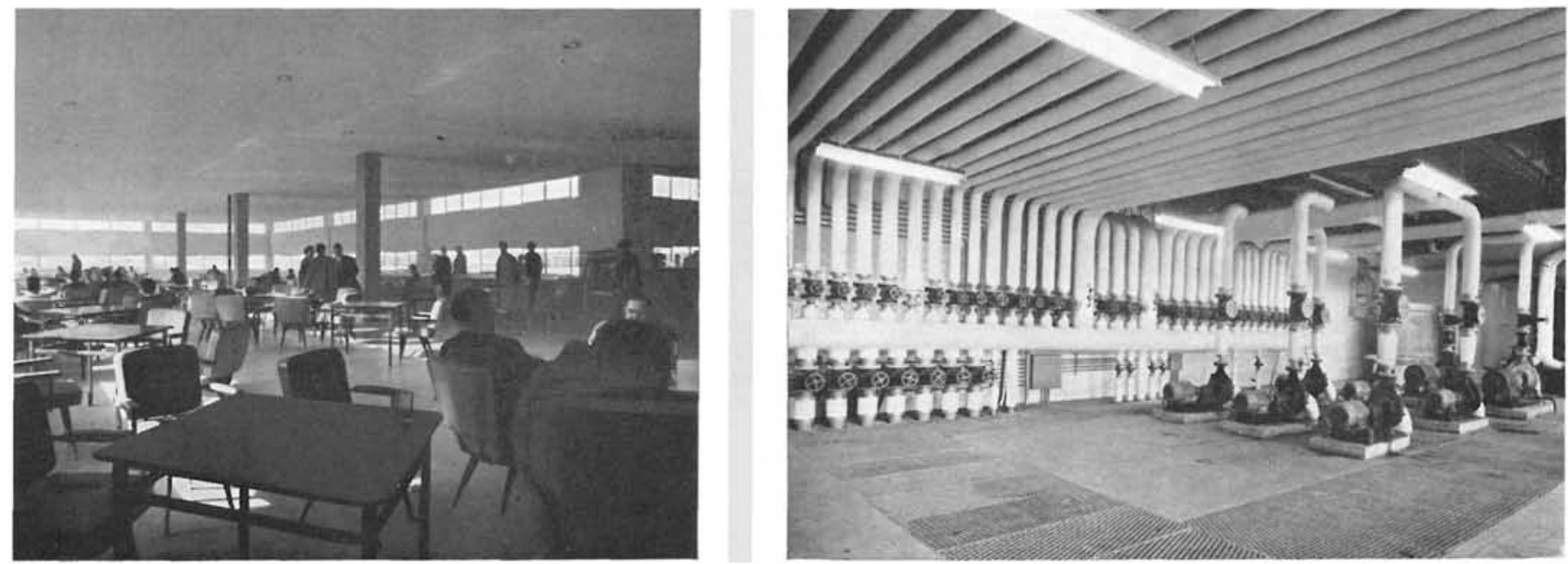

Foton gENJAMIN GUTIERREZ y PAISAJES ESPAÑOLES

Las zonas de poniente y saliente, con amplios accesos, se ocupan con aulas y dependencias docentes y de uso general, quedando entre ambas el Aula Magna.

En la parte sur se dispone el área de alumnos con capilla, sala de estar, autoservicio, club, TEU, etc.

Los laboratorios de prácticas quedan inmediatamente debajo de los núcleos respectivos a los que sirven y en comunicación directa con los mismos.

Dentro de los núcleos de aulas, antes del cambio de plan estaban separadas éstas por cursos, con sus vestíbulos de espera propios.

La concentración de la planta excusó de duplicar algunos servicios, propuestos separados; en efecto, parece suficiente construir una sola capilla, si ésta queda inmediata a todos los cursos, como se ha conseguido en nuestro caso.

Con mayor razón estimamos de interés instalar una sola cafetería, próxima a los grupos de aulas, al salón de actos y al club, no solamente por el costo inicial de instalación, sino por el mantenimiento, personal, cámaras, almacenes, etc.

Por la disposición adoptada, todos los grupos quedan relacionados entre sí, a la vez que con la debida independencia, de tal forma que podríamos hacer funcionar una sola de las secciones o núcleos sin interferir a las demás, con sus entradas y servicios propios o, por el contrario, controlar todos los grupos haciéndolos pasar por el vestíbulo general.

La colocación de seminarios responde a la relación que tienen con el resto del edificio. Hemos buscado un emplazamiento que quede inmediato a los núcleos de alumnos, a la dirección, a la biblioteca a la

EI sistema empleado para el forjado ha sido el de losa aligerada nervada en dos direcciones. Toda la estructura estaba modulada en EI Sistema empleado para el forjado ha sido el de losa aligerada nervada en dos
$8 \times 8 \mathrm{~m}$ o en múltiplos de $8 \mathrm{~m}$, lo que facilitó el cálculo y la ejecución del forjado.

Para aligerar la losa nervada en dos direcciones se emplearon bloques de poliéster reforzado de fibra de vidrio, según patente del Dr. ingeniero industrial D. Joaquín Roura. Estos bloques se emplearon como encofrado apoyados sobre tablones.

La colocación de los bloques es muy sencilla y una vez colocados queda preparado el encofrado, tanto de las nervaduras longitudinales (formados por los tablones) como de las transversales; estas últimas formadas por acoplamiento de dos bloques.

La recuperación de los bloques se puede efectuar a las 48 horas de hormigonada la losa, toda vez que para su recuperación no es preciso retirar tablones ni puntales, los cuales permanecen durante el tiempo reglamentario de fraguado. Elo permitió realizar la obra con vado (cerca de 90),

En la foto adjunta se puede observar la ligereza de los bloques, que facilita su manejo; así como su enorme rigidez y resistencia a la compresión.

En la propia obra se montó un pequeño taller de reparación de bloques, atendido por un peón especializado. En dicho taller se procedia a la reparación de aquellos bloques que hacía empleando tela de fibra de vidrio y poliéster.

El costo total de amortización de bloques y entretenimiento y reparación de los mismos resultó a 32 pesetas por metro cuadrado de forjado.

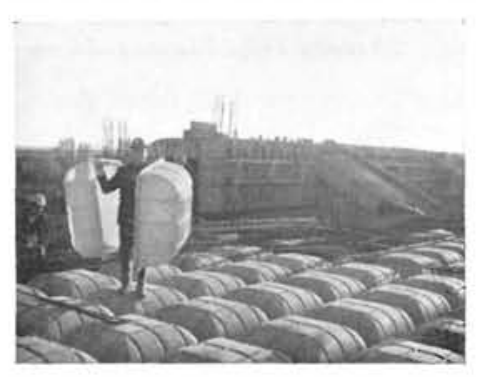


sección de publicaciones, porque entendemos que con todos debe mantener íntimo contacto. Dado el número de ellos y su programa, en vertical se puede resolver fácilmente el problema de su emplazamiento, lo cual no resulta insólito, ya que es usual resolver en torre los edificios de este tipo, como en los laboratorios de investigación de Wisconsin, de Frank Lloyd Wright.

Por otra parte, la construcción de una torre tiene grandes ventajas en cuanto a composición, no solamente porque centra y equilibra las masas de las diferentes plataformas, sino porque estando este edificio en una cota muy por debajo de la que corresponde a la Facultad de Filosofía y Letras, necesita de un volumen dominante para no quedar coronado por el de aquella Facultad.

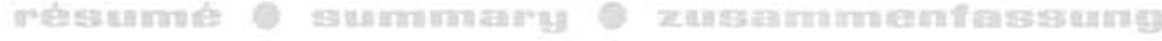

\section{Ecole Technínue Supémieure des Ingénieurs des Ponts et

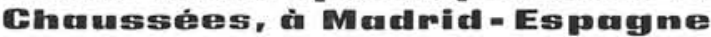

Luis Laorga et José López Zanón, architectes

Le projet a remporté le premier prix d'un concours convoqué à cet effet en 1963. Il a été résolu en accord avec les normes universellement acceptées de dimensionnement correct, d'éclairage uniforme, de ventilation transversale, de réduction de niveaux, de concentration maximale et de circulations courtes, d'organisation hiérarchique des différents noyaux et fonctions, de centralisation des zones de détente par rapport à leurs groupes correspondants.

L'édifice a été le résultat d'une construction soignée, pour laquelle ont été utilisés fondamentalement des matériaux durables et de facile entretien, tels que le béton apparent et l'aluminium anodisé.

\section{Higher technical school of civil engimeering in Meadrid, Sperim}

Luis Laorga \& José López Zanón, architects

The project was awarded the first price at a competition initiated in 1963, and it is based on the universally establis. hed practice regarding careful and strict dimensioning, uniform illumination, transversal ventilation, low ceilings, maximum concentration and short circulation distances, as well as coordinated organization of the various nuclei and functions, and centralization of the relaxation zones.

The actual building has been carefully constructed, with long lasting materials requiring little maintenance, such as unfaced concrete and anodized aluminium.

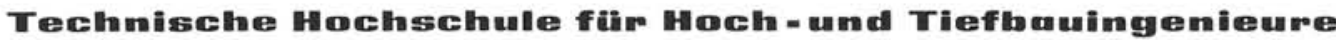

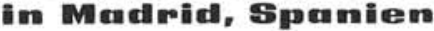

Luis Laorga und José López Zanón, Architekten

Dem Entwurf wurde einem im Jahre 1963 stattgefundenen Wettbewerb der erste Preis verliehen. Er richtet sich nach den allgemein geltenden Prinzipien: geeignete und genaue Dimensionierung, gleichmässige Beleuchtung, Querlüftung, und Höchstmass an Konzentrierung, um die Etagenanzahl und die Verkehrsflächen zu reduzieren, sowie rich. tige Plazierung der Freizeiträume innerhalb der entsprechenden Gruppen.

Dieses Gebäude ist das Ergebnis einer sorgfältigen Ausführung, bei der grundsätzlich dauerhafte und leicht zu unterhaltende Materialien, wie Sichtbeton und wetterfestes Aluminium verwendet wurden. 